\title{
Percepción de la calidad en Centros de Atención Temprana: Resultados de un análisis comparativo
}

\section{Perceived Quality in Early Intervention Centres: Results of a Comparative Analysis}

\author{
Rita Pilar Romero-Galisteo ${ }^{1,2}$, Pablo Gálvez-Ruiz ${ }^{3}$, Juan Carlos Belda Oriola ${ }^{4}$, \\ Maria Gracia E. Millá-Romero ${ }^{5}$
}

${ }^{1}$ Centro de Atención Temprana del Excmo. Ayto. de Antequera, Málaga, España. ${ }^{2}$ Universidad de Málaga. Facultad Ciencias de la Salud. Dpto. de Fisioterapia, España. ${ }^{3}$ Universidad Internacional de La Rioja, España. ${ }^{4}$ Consellería Sanidad Comunitat Valenciana, España. ${ }^{5}$ Centro de Atención Temprana APADIS. Villena, España.

Disponible online 31 de agosto de 2015

La Atención Temprana es considerada actualmente como un tipo de intervención imprescindible para promover el desarrollo de niños con trastornos en su desarrollo, o riesgo de padecerlos, y de sus familias. El objetivo de este trabajo fue analizar la percepción de la calidad del servicio prestado en los Centros de Atención Temprana por parte de los usuarios. Participaron 397 familias que recibían atención temprana en dos centros diferentes donde se administró el Inventario de Calidad en Centros de Atención Infantil Temprana. Los resultados muestran que, tanto en el primer estudio como en el segundo, los usuarios de ambos centros valoraron positivamente el servicio que habían recibido, siendo las dimensiones Personal Especializado $(M=4.77, D T=0.48)$ e Información Técnica $(M=4.54, D T=0.70)$ las que obtuvieron valores medios más elevados. Se encontraron diferencias significativas entre ambos estudios en las dimensiones Instalaciones del centro $(M=4.39, D T=0.41)$ y Salas de tratamiento $(M=4.66, D T=0.55)$ de uno de los centros. La evaluación de la calidad percibida por parte de las familias atendidas en los Centros de atención temprana sirve para detectar debilidades a mejorar y fortalezas a mantener en el contexto de mejora continua instaurado en los servicios de atención temprana.

Palabras Clave: Atención temprana; Calidad Percibida; Familia.

Early Intervention is currently regarded as an essential strategy to promote the development of children with developmental disorders, or who are at risk of them, and to promote the wellbeing of their families. This article analysed perceived service quality in Early Intervention Centres. The sample comprised 397 families receiving Early Intervention at two centres, where they were administered the Early Intervention Centre Quality Inventory. The results of both studies suggest that the users of both centres positively appraised the service they had received: the dimensions Qualified Staff $(M=4.77, S D=0.48)$ and Technical Information $(M=4.54, S D=0.70)$ obtained the highest mean values. There were significant differences between the studies in the dimensions Facilities $(M=4.39, S D=0.41)$ and Treatment Rooms $(M=4.66, S D=0.55)$. The evaluation of the perceived quality by the families attending Early Intervention Centres serves to identify and weaknesses to be improved and strengths to be maintained in a context of the continuous improvement established in these kinds of services.

Key Words: Early Intervention; Perceived Quality; Families.

Correspondencia: Rita Pilar Romero-Galisteo. Departamento de Fisoterapia. Facultad de Ciencias de la Salud. C/ Arquitecto Francisco Peñalosa. Ampliación del Campus de Teatinos. Universidad de Málaga. 29071-Málaga. E-mail: rpromero@uma.es. E-mails de los co-autores: Pablo GálvezRuiz: pablo.galvez@unir.net; Juan Carlos Belda Oriola: beldhern@gmail.com, Maria Gracia E. Millá-Romero: atenciontemprana@apadis.com 
La evolución de la Atención Temprana (AT) a lo largo de sus escasos cincuenta años de historia ha permitido que actualmente sea considerada como un tipo de intervención imprescindible para promover el desarrollo y la salud de niños con trastornos en su desarrollo, o riesgo de padecerlos, y de sus familias.

Los motivos de este tipo de intervención tendríamos que buscarlos, según Pérez-López (2014) en las bases teóricas que imperaban en la Psicología a mediados del S.XX. Los programas de intervención estaban basados en modelos clínicos, en los que se trataba de recuperar o rehabilitar las áreas afectadas del niño. La familia jugaba un papel pasivo y el profesional era concebido como un experto. Esta intervención se realizaba sobre los niños que presentaban patologías establecidas desde el momento del nacimiento hasta los tres años de vida. En esos años, a este campo de actuación se le denominaba estimulación precoz y solo se prestaba asistencia a personas con discapacidad. En los últimos 30 años se ha ido sustituyendo este modelo meramente asistencial por otro de carácter preventivo (Pons, 2007). Por tanto, no se trata de intentar evitar que déficits biológicos, psicológicos y sociales interfieran en la vida del niño sino de evitar su aparición o actuar sobre factores biológicos o situaciones de privación socio-ambiental que pudieran provocar la aparición de dichos déficits. Comienza entonces a trabajarse desde una perspectiva multidisciplinar y con la firme creencia de que los niños con estos déficits pueden desarrollar una vida útil e integrarse perfectamente en la sociedad, siempre y cuando reciban las atenciones específicas y necesarias (Robles-Bello y Sánchez-Teruel, 2013).

Diferentes modelos teóricos han servido, por tanto, de sostén a la AT. Desde el Modelo Transaccional propuesto por Sameroff y Chandler (1975), a la Teoría de los Sistemas Ecológicos de Bronfenbrenner (1979), pasando por el Modelo de los Sistemas Evolutivos (Guralnick, 2000, 2005, 2011) o el Modelo Transdisciplinar (King et al., 2009), la AT ha ido evolucionando y haciendo cada vez más partícipe a la familia, considerándola como uno de los pilares sobre los que debe basarse toda actuación (Candel, 2005; De Linares y Rodríguez, 2004; McWilliam, 2010).

Actualmente, los primeros años de vida del ser humano siguen considerándose como una etapa crucial en la maduración del individuo. El estudio de esta primera infancia ha determinado la importancia de diferentes intervenciones sistémicas y multidisciplinares. Si bien estas actuaciones resultan imprescindibles cuando aparece una alteración del desarrollo, no debemos olvidar el protagonismo esencial del entorno familiar y el contexto natural que rodea al niño (Palisano et al., 2011; Peterander, 2009).

Con la publicación en España y en otros países del Libro Blanco de la AT se unificaron criterios conceptuales, entendiéndose la AT como:

Un conjunto de intervenciones dirigidas a la población infantil de 0-6 años, a la familia y al entorno, que tiene por objetivo dar respuesta lo más pronto posible a las necesidades tran- sitorias o permanentes que presentan los niños con trastornos en su desarrollo o que tienen riesgo de padecerlos. Estas intervenciones, que deben considerar la globalidad del niño, han de ser planificadas por un equipo de profesionales de orientación interdisciplinar o transdisciplinar (GAT, 2000, 2005, p. 13).

Por otro lado, los cambios organizativos acaecidos en nuestra sociedad han aumentado el interés por medir la calidad de los servicios que reciben los ciudadanos. La AT en nuestro país se presta tanto en el ámbito sanitario, como social y educativo siendo los Centros de Desarrollo Infantil y Atención Temprana (CDIAT), servicios de carácter interdisciplinar donde se atiende a población entre cero y seis años que presentan alguna alteración o disfunción en su desarrollo o riesgo de padecerla (Bugié, 2002). Este tipo de centros donde se presta AT es el objeto de nuestra investigación y, como servicio que es, tienen componentes intangibles que hacen necesario tener en cuenta la percepción subjetiva del cliente para evaluar la calidad (Saurina, 1997), en este caso, las familias de los niños y niñas atendidas en los CDIAT.

Parasuraman, Zeithalm y Berry $(1985,1988)$ y Parasuraman, Berry y Zeithalm (1991) definen la calidad percibida como la diferencia entre las expectativas y las percepciones de los consumidores respecto a un servicio. Estudios posteriores pusieron de manifiesto que los encuestados no distinguían entre ambos conceptos (McAlexander et al., 1994; Saurina y Coenders, 1999). También se ha sugerido que no es necesario preguntar por las expectativas de forma separada (Cronin y Taylor, 1994). El único nexo en común de la línea de investigación sobre la medición de la calidad en el sector servicios ha sido el empleo de encuestas y tests. Las aplicaciones psicológicas a datos procedentes de tests y las sociológicas y politológicas a datos procedentes de encuestas fueron posiblemente las pioneras, y siguen siendo las más numerosas, pues tanto los tests como las encuestas suelen contener gran número de variables (Batista y Coenders, 2012) que, según el constructo a investigar, podrán construirse específicamente para ser utilizados en un contexto determinado.

En las organizaciones, la concepción de calidad de servicio varía de unos grupos de interés a otros. En las que se dedican a prestar servicios a personas con alteraciones del desarrollo, los profesionales que trabajan en los centros tienen su propia concepción de calidad de servicio que se basa, en buena medida, en criterios profesionales adquiridos y en las trayectorias y experiencias profesionales. Los usuarios, en este caso, las familias, no tienen por qué compartir esa concepción, así como los propios gerentes y directores de los centros (Martínez-Tur, 2011).

La calidad se plantea como una ventaja competitiva de la empresa ante su competencia, en la medida que se presenta como garantía para el cliente, y esa resulta ser la razón esencial para su implantación cada vez más generalizada. Esta ventaja en los CDIAT se transforma en la justificación de los fondos públicos utilizados ante la administración competente (Ponte, Cardama, Arlanzón, Belda, González y Vived, 2008). 
Siendo conscientes de los inconvenientes y problemas que, a nivel metodológico han presentado los instrumentos de medida propuestos hasta la fecha, Milla (2004) plantea la necesidad de diseñar cuestionarios y formular encuestas, cumplimentados de manera anónima, que permitan estudiar todos los indicadores con los que se realice la evaluación interna de los puntos fuertes y débiles en los servicios de AT. Se planteó entonces el diseño y validación de una herramienta creada ad hoc para medir la calidad de servicio percibida por parte de las familias atendidas en los Centros de Atención Infantil Temprana (Romero-Galisteo, Morales-Sánchez y Hernández-Mendo, 2015).

Una de las propuestas de Martínez-Tur, Peiró, Moliner y Potonick (2010) es utilizar la retroalimentación a través de datos de encuesta orientada a la mejora de la calidad de servicio. Este proceso de transferencia ha servido para plantear nuevos objetivos en nuestra línea de investigación.

Analizar la calidad del servicio prestado en los Centros de Atención Temprana mediante el uso de una herramienta validada, Inventario de Calidad en Centros de Atención Infantil Temprana (ICCAIT), fue el objetivo general del estudio que aquí se presenta. Determinar si se han mantenido los puntos fuertes y si han mejorado las debilidades detectadas con esta herramienta con el fin de instaurar una filosofía de Calidad Total en los centros participantes fue el objetivo secundario de nuestra investigación.

\section{Participantes \\ Método}

La muestra total se compuso de 397 usuarios procedentes de dos CDIAT de la provincia de Málaga, de los cuales 285 eran mujeres y 83 hombres, dejando 29 participantes sin responder esta pregunta. En cuanto a la edad, el $54.9 \%$ de la muestra estaba comprendida entre 30 y 39 años, el $18.6 \%$ entre 40 y 49 años y el $14.90 \%$ entre 20 y 29 años. Se registraron porcentajes muy bajos tanto de menores de 20 años $(0.80 \%)$ como mayores de $50(0.60 \%)$, además de contar con un $9.80 \%$ de valores perdidos. Se realizó un muestreo no aleatorio por selección intencionada donde los criterios de inclusión fueron que las familias decidieran participar de manera anónima y voluntaria y que llevaran más de un mes asistiendo a sesiones de AT, para que conocieran el funcionamiento del centro. Como único criterio de exclusión se estableció que los participantes no comprendieran o pudieran expresarse en castellano.

\section{Instrumento}

El instrumento utilizado fue el Inventario de Calidad en los Centros de Atención Infantil Temprana (ICCAIT) de RomeroGalisteo, Morales-Sánchez y Hernández-Mendo (2015). Se trata de una batería de 48 ítems, encabezada por una serie de variables sociodemográficas, utilizada para evaluar la calidad percibida por los usuarios del servicio de atención temprana a partir de seis dimensiones: Instalaciones, Salas de Tratamiento y Material, Atención al Usuario, Personal Especializado, Información
General e Información Técnica. Las respuestas mostraban un rango de puntuación tipo Likert que oscila entre 1 (totalmente en desacuerdo) y 5 (totalmente de acuerdo). Se optó por eliminar la dimensión Información General debido a la baja fiabilidad obtenida en el estudio original $(\alpha=.32)$ y a los pobres resultados obtenidos en el análisis factorial exploratorio, cuya pertinencia mostró un índice KMO de .52 y un determinante de .86 .

En este estudio, la fiabilidad global para la escala fue adecuada $(\alpha=.94)$, obteniéndose alfas de $.84, .91, .76, .92, .88$ para las cinco dimensiones, respectivamente, siendo por tanto muy similares a las obtenidas en el estudio original y acordes a los valores recomendados (Nunnally, 1978).

\section{Procedimiento}

La herramienta se pasó en dos periodos, noviembre de 2011 y enero de 2014, a los dos CDIAT de manera simultánea, de manera que en el primer estudio respondieron al cuestionario 177 personas (86 al CDIAT-A y 91 al CDIAT-B) mientras que en el segundo fueron un total de 220 (100 al CDIAT-A y 120 al CDIAT-B).

El cuestionario fue administrado por dos investigadores en los dos CDIAT participantes, donde los familiares de los niños que recibían atención temprana respondieron a los ítems de manera individual, voluntaria y totalmente confidencial. Previamente se les recordó que no existían respuestas correctas o incorrectas, solicitándoles que participaran con total honestidad. Ante cualquier tipo de ayuda o duda, se les indicó que acudieran a los investigadores, presentes en una sala contigua para proporcionar la información necesaria para la correcta cumplimentación del cuestionario.

\section{Análisis de datos}

Una vez codificados los datos, se realizaron varios análisis con el paquete estadístico SPSS 21.0 referidos a datos descriptivos, consistencia interna y correlación entre las dimensiones en los distintos estudios.

\section{Resultados}

Los análisis descriptivos y correlaciones entre dimensiones se presentan en la tabla 1 , donde se aprecia un valor medio entre 4.19 y 4.77 , lo que indica una buena valoración en términos generales del servicio. El resultado más elevado se encuentra en la dimensión Personal Especializado $(M=4.77 ; D T=.48)$, seguida de Información Técnica $(M=4.54$; DT $=.70)$, mientras que en el extremo contrario, la Instalación representa la dimensión con menor valoración $(M=4.19 ; D T=.63)$. Todo ello parece indicar que los usuarios del servicio muestran mayor interés en los aspectos relacionados con la especialización y profesionalidad del equipo multidisciplinar existente en los centros de atención temprana, además de todo lo relacionado con las actividades que proponen para realizar, la coordinación entre los distintos componentes del equipo, el seguimiento, la progresión y el plan de intervención realizado. 
Todas las dimensiones correlacionan significativamente, lo que indica que todas ellas analizan un constructo muy próximo. La relación más elevada se obtiene en el par Instalaciones y Salas de Tratamiento y Material $(r=.80, p=.000)$, lo que implica que los usuarios consideran que la instalación resulta adecuada teniendo en cuenta que las salas de tratamiento y material es lugar donde se realiza la intervención directa tanto con la familia como con el niño.

Tabla 1

Análisis descriptivos de las dimensiones y correlaciones entre las mismas.

\begin{tabular}{lccccc}
\hline \multicolumn{1}{c}{ Dimensiones } & 1 & 2 & 3 & 4 & $6^{\mathrm{a}}$ \\
\hline $\begin{array}{l}\text { 1. Instalaciones } \\
\begin{array}{l}\text { 2. Salas tratamiento } \\
\text { y material }\end{array}\end{array}$ & -- & $.80^{* *}$ & $.46^{* *}$ & $.50^{* *}$ & $.38^{* *}$ \\
$\begin{array}{l}\text { 3. Atención al } \\
\text { usuario }\end{array}$ & -- & $.55^{* *}$ & $.57^{* *}$ & $.43^{* *}$ \\
$\begin{array}{l}\text { 4. Personal } \\
\text { especializado }\end{array}$ & & & -- & $.62^{* *}$ & $.64^{* *}$ \\
$\begin{array}{l}\text { 6. Información } \\
\text { técnica }\end{array}$ & & & & $.69^{* *}$ \\
\hline $\begin{array}{l}\text { Media } \\
\text { Desviación típica }\end{array}$ & .63 & .67 & .62 & .48 & .70 \\
\hline
\end{tabular}

Nota. ${ }^{a}$ Los números se corresponden con los de las dimensiones en la primera columna. $*_{p}^{*}<.01$

En cuanto a las diferencias existentes en el CDIAT-A en la percepción de las dimensiones de calidad se pueden apreciar en la tabla 2, donde observamos que las valoraciones son superiores en el segundo estudio en todas las dimensiones con excepción de Información Técnica $(M=4.41, D T=0.78)$ y Personal Especializado, que obtuvo prácticamente el mismo valor medio. Las diferencias más altas se encontraron en las dimensiones Instalaciones (.68) y Salas de Tratamiento y Material (.62). Se obtuvieron diferencias en el orden, aunque en ambos estudios el Personal Especializado fue la dimensión más valorada; la Sala de Tratamiento y Material se situó como la segunda dimensión mejor valorada en el segundo estudio y la información técnica, pese a situarse en cuarto lugar no obtuvo una gran diferencia en la valoración media. Estas diferencias han resultado ser significativas tan sólo en la dimensión Instalaciones $(M=4.39, D T=$ $.41, t(128)=7.62, p<.01)$ y Salas de Tratamiento y Material $(M=$ $4.66, D T=.55, t(156)=6,53, p<.01)$

Tabla 2

Comparación en la percepción de las dimensiones de la calidad en el CDIAT-A.

\begin{tabular}{|c|c|c|c|c|c|c|c|c|}
\hline & \multicolumn{3}{|c|}{ Noviembre 2011} & \multicolumn{3}{|c|}{ Enero 2014} & \multirow[b]{2}{*}{$t$} & \multirow[b]{2}{*}{$p$} \\
\hline Variables & $n$ & $M$ & $D T$ & $n$ & $M$ & $D T$ & & \\
\hline Instalaciones & 86 & 3.70 & .73 & 100 & 4.39 & .41 & 7.62 & $.000 *$ \\
\hline $\begin{array}{l}\text { Salas de trat. y } \\
\text { material }\end{array}$ & 86 & 4.03 & .73 & 100 & 4.66 & .55 & 6.53 & $.000^{*}$ \\
\hline $\begin{array}{l}\text { Atención al } \\
\text { usuario }\end{array}$ & 86 & 4.37 & .61 & 100 & 4.43 & .67 & .60 & .549 \\
\hline $\begin{array}{l}\text { Personal } \\
\text { especializado }\end{array}$ & 86 & 4.72 & .51 & 100 & 4.73 & .56 & .137 & .892 \\
\hline $\begin{array}{l}\text { Información } \\
\text { técnica }\end{array}$ & 86 & 4.43 & .71 & 100 & 4.41 & .78 & -.177 & .861 \\
\hline
\end{tabular}

Nota: $*_{p}<0.01$
En cuanto al CDIAT-B (tabla 3), las valoraciones en el primer estudio coinciden en orden con las obtenidas también para el primer estudio del CDIAT-A, aunque en este centro prácticamente no hubo variación del primer al segundo estudio. Tan sólo la dimensión Salas de Tratamiento y Material intercambió el orden con la dimensión Atención al Usuario. Para este centro, las dimensiones relacionadas con los aspectos tangibles fueron las únicas que mejoraron en el segundo estudio, mientras que las valoraciones medias para las dimensiones Personal Especializado, Información Técnica y Atención al Usuario fueron inferiores. Pese a esto, ninguna de las diferencias en la percepción del servicio resultó significativa $(p>.01)$.

Tabla 3

Comparación en la percepción de las dimensiones de la calidad en el CDIAT-B.

\begin{tabular}{lcccccccc}
\hline & \multicolumn{1}{c}{ Noviembre 2011} & \multicolumn{1}{c}{ Enero 2014} \\
\hline \multicolumn{1}{c}{ Variables } & $n$ & $M$ & $D T$ & $n$ & $M$ & $D T$ & $t$ & $p$ \\
\hline Instalaciones & 91 & 4.22 & .53 & 120 & 4.33 & .61 & 1.34 & .179 \\
Salas de tratamiento & 91 & 4.33 & .64 & 120 & 4.48 & .64 & 1.71 & .088 \\
y material & & & & & & & & \\
Atención al usuario & 91 & 4.38 & .47 & 120 & 4.38 & .68 & .006 & .995 \\
Personal especializado & 91 & 4.86 & .24 & 120 & 4.79 & .52 & -1.29 & .196 \\
Información técnica & 91 & 4.66 & .59 & 120 & 4.64 & .66 & -.32 & .748 \\
\hline
\end{tabular}

Los resultados obtenidos implican que en ambos centros, cuando valoran la calidad del servicio perciben como indicadores básicos aquellos elementos intangibles referidos al personal especializado que trabaja en el mismo, la información técnica que proporcionan y la atención al usuario. Los elementos tangibles, como son las salas de tratamiento y material así como las instalaciones, se sitúan en último lugar, aunque las valoraciones obtenidas en el segundo estudio del CDIAT-A mostraron diferencias significativas con respecto al primer estudio, demostrando así que se han introducido elementos que los usuarios interpretan como mejoras en el propio servicio.

\section{Discusión}

Los resultados que se obtienen en esta investigación permiten afirmar que las familias perciben globalmente un nivel de calidad elevado en los CDIAT a los que acuden. Esta es una conclusión relevante ya que estudios como éste hacen que, en la gestión de los servicios de AT, se vaya instaurando un sistema de gestión de la Calidad Total.

Las situaciones y cambios que se han producido en nuestra sociedad a lo largo de las últimas décadas han permitido configurar las respuestas que la sociedad ha ofrecido tanto a la infancia como a los sujetos con discapacidad, según el momento histórico y filosófico (Gútiez y Ruiz, 2012) y la gestión de la calidad en los servicios de AT ha sido uno de los retos más importantes que se están planteando actualmente.

Los Centros de Atención Temprana, como organizaciones donde se prestan servicios de AT están implantando nuevas fórmulas para su gestión. Como apunta Martínez-Tur (2011), internamente, las organizaciones pueden establecer procedimientos y cauces para la mejora continuada en la calidad de ser- 
vicio; externamente, se pueden establecer lazos de cooperación con centros y organizaciones de generación del conocimiento (p.e: universidades o empresas especializadas). En consonancia con la transferencia de los resultados de la investigación a la sociedad que se preconiza desde diferentes sectores, el survey-feedback, cuya traducción resumida sería la de "retroalimentación a través de datos de encuesta", es una metodología clásica de cambio organizativo (Martínez-Tur, Peiró, Moliner y Potonick, 2010; Peiró, González-Romá y Cañero, 1999) que ha sido utilizada en este estudio con el fin de mejorar la calidad del servicio prestado en los CDIAT. Utilizar un procedimiento de retroalimentación de la información obtenida mediante las herramientas de evaluación creadas ad hoc y validadas para tal fin, resulta práctico y útil para proponer medidas de mejora en la calidad asistencial, tanto para el gestor como para los profesionales de la AT y esta filosofía de Calidad Total, redundará positivamente en las familias que reciben atención en este tipo de centros.

Los resultados obtenidos muestran que las familias aprecian positivamente las dimensiones intangibles, como la información técnica recibida por parte de los profesionales que integran el equipo de AT que atiende a su hijo-a, coincidiendo este aspecto con el estudio de Martínez-Fuentes y MartínezHernández (2013). La atención al usuario prestada por el personal del centro representa igualmente una dimensión relevante en ambos estudios. En cuanto al personal especializado, la adecuada capacitación profesional es un aspecto que alcanza una valoración media elevada en los dos estudios realizados en ambos centros. En cuanto a los aspectos tangibles del servicio, ambos centros obtuvieron las valoraciones medias más bajas para los dos estudios tanto para la dimensión salas de tratamiento y material como para instalaciones, aunque en el caso del CDIAT-A se obtuvieron mejoras significativas en el segundo estudio. De estos resultados se desprenden una serie de pautas que pueden ser relevantes en la comprensión del servicio y en el esfuerzo por la optimización de su excelencia.

Una limitación del estudio es el escaso número de centros analizados por lo que, a pesar de obtener conclusiones alentadoras, solo reflejan la realidad, difícilmente generalizable, de dos CDIAT. En un futuro, por tanto, se propone analizar datos provenientes de un mayor número de familias y centros, pudiendo resultar interesante el análisis de la calidad percibida en función de los distintos modelos de financiación que aún persisten en España. Coincidiendo con Belda (2008), se antoja necesario la evaluación externa del constructo calidad en los CDIAT, de cara a implantar un modelo de Calidad Total en este tipo de servicios, por lo que, desde aquí se recomienda la realización de este tipo de estudios utilizando las herramientas y metodología adecuadas.

\section{Referencias}

1. Belda, J. C. (2008). Evaluación externa de calidad de un centro de desarrollo infantil y atención temprana. Siglo Cero, 39, 5-17.
2. Bronfenbrenner, U. (1979). The ecology of human development. Cambridge: Harvard University Press.

3. Batista, J. M. y Coenders, G. (2012). Modelos de ecuaciones estructurales. Madrid: La Muralla.

4. Bugié, C. (2002). Centros de desarrollo infantil y Atención Temprana. Revista de Neurología, 34, 139-143.

5. Candel, I. (2005). Elaboration of a program of early intervention. Electronic Journal of Research in Educational Psychology, 3, 151-192.

6. Cronin, J. J. y Taylor, S. A.(1994). SERVPERF versus SERVQUAL: reconciling performance-based and perceptions-minus-expectatios measurement of service quality. Journal of Marketing, 56, 125-131. http://dx.doi. org/10.2307/1252256

7. De Linares, C. y Rodríguez, T. (2004). Bases de la atención familiar en la Atención Temprana. En J. Pérez-López y A. G. Brito (Eds.), Manual de Atención Temprana (pp. 333351). Madrid: Pirámide.

8. Grupo de Atención Temprana [GAT] (2000). Libro Blanco de la Atención Temprana. Madrid: Real Patronato sobre Discapacidad.

9. Grupo de Atención Temprana [GAT] (2005). White paper on early intervention. Madrid: Real Patronato sobre Discapacidad.

10. Guralnick, M. J. (2000). Interdisciplinary team assessment for young children: purposes and processes. En M. J. Guralnick (Ed.), Interdisciplinary clinical assessment of young children with developmental disabilities (pp. 3-15). Baltimore: Paul Brookes Publishing.

11. Guralnick, M. J. (2005). Early intervention for children with intellectual disabilities: current knowledge and future prospects. Journal of Applied Research in Intellectual Disabilities, 18, 313-324. http://dx.doi.org/10.1111/j.14683148.2005.00270.x

12. Guralnick, M. J. (2011). Why early intervention works: A systems perspective. Infants \& Young Children, 24, 6-28. http://dx.doi.org/10.1097/IYC.0b013e3182002cfe

13. Gutiez, P. y Ruiz, E. (2012). Orígenes y evolución de la Atención Temprana. Una perspectiva histórica de la génesis de la Atención Temprana en nuestro país. Agentes, contextos y procesos. Psicología Educativa, 18, 107-122. http:// dx.doi.org/10.5093/ed2012a12

14. King, G., Strachan, D., Tucker, M., Duwyn, B., Desserud, S. y Shillington, M. (2009). The application of a transdisciplinary model for early intervention services. Infants \& Young Children, 22, 211-223. http://dx.doi.org/10.1097/ IYC.0b013e3181abe1c3

15. McAlexander, J. H., Kaldenburg, D. O. y Koening, H. F.(1994). Service quality measurement. Journal of Health Care Marketing, 14, 34-40.

16. McWilliam, R. A. (2010). Routines-based early intervention-supporting young children and their families. Maryland: Paul H Brookes Publishing Company.

17. Martínez-Fuentes, M. T. y Martínez-Hernández, L. (2013). 
Promoción del desarrollo infantil y atención temprana: calidad de los servicios. Azarbe: Revista internacional de trabajo social y bienestar, 2, 49-67.

18. Martínez-Tur, V., Peiró, J. M., Moliner, C. y Potonick, C. (2010). Calidad de servicio y calidad de vida: El "surveyfeedback" como estrategia de cambio organizacional. Madrid: FEAPS

19. Martínez-Tur, V. (2011). El sueño de la calidad de servicio. Siglo Cero, 42, 24-30.

20. Milla, M. G. (2004). La calidad en los servicios de atención temprana: una propuesta de futuro. En J. Pérez-López y A. G. Brito (Eds.), Manual de Atención Temprana (pp. 369380). Madrid: Pirámide

21. Nunnally, J. C. (1978). Psychometric Theory. Nueva York: McGraw-Hill.

22. Palisano, R. J. Chiarello, L. A., King, G. A., Novak, I., Stoner, T. y Fiss, A. (2012). Participation-based therapy for children with physical disabilities. Disability \& Rehabilitation, 34, 1041-1052. http://dx.doi.org/10.3109/09638288.2 $\underline{011.628740}$

23. Parasuraman, A., Zeithalm, V. A. y Berry, L. L. (1985). A conceptual model of service quality and its implications for future research. Journal of Marketing, 49, 41-50. http:// dx.doi.org/10.2307/1251430

24. Parasuraman, A., Zeithalm, V. A. y Berry, L. L. (1988). SERVQUAL: a multiple-item scale for measuring consumer percepcions of service quality. Journal of Reatiling, 64, 41-50.

25. Parasuraman, A., Berry, L. L. y Zeithalm, V. A. (1991). Refinement and reassessment of the SERVQUAL scale. Journal of Reatiling, 67, 420-450.

26. Peiró, J., González-Romá, V. y Cañero, J. (1999). Survey feedback as a tool for changing managerial culture. Focusing on user's interpretations. A case study. European Journal of Work and Organizational Psychology, 8, 537-550. http://dx.doi.org/10.1080/135943299398159
27. Pérez-López, J. (2014). Marco teórico en atención temprana y nuevas necesidades de formación para una intervención eficaz. En J. Piñero, J. Pérez-López, F. Vargas y A.B. Candela (Coords.), Atención temprana en el ámbito hospitalario. Madrid: Editorial Pirámide.

28. Peterander, F. (2009). Influencia del entrono en la salud mental infantil e intervención temprana. http://www. genysi.es/index.php?option $=$ com_remository\&Itemid $=32$ \&func=fileinfo\&id=334 (consultado en enero de 2015)

29. Pons, A. (2007). Atención Temprana en Andalucía. Vox Paediatrica, 15, 26-29.

30. Ponte, J., Cardama, J., Arlanzón, J. L., Belda, J. C., González, T. y Vived, E. (2008). Guía de estándares de calidad en Atención Temprana. Madrid: Real Patronato sobre Discapacidad.

31. Robles-Bello, M. A. y Sánchez-Teruel, D. (2013). Atención infantil temprana en España. Papeles del Psicólogo, 34, 132-143.

32. Romero-Galisteo, R. P., Morales-Sánchez, V. y HernándezMendo, A. (2015). Desarrollo de una herramienta para medir la calidad percibida en los centros de atención infantil temprana. Anales de Psicología, 31, 127-136. http:// dx.doi.org/10.6018/analesps.31.1.158191

33. Saurina, C. (1997). Evaluación de un modelo de medida de la calidad en el sector servicios. Las entidades financieras en las comarcas de Girona. Estadística española, 39, 219248.

34. Saurina, C. y Coenders, G. (1999). Predicting overall service quality. A structural equation modelling approach. Comunicación presentada a la Conferencia Internacional de Metodología y Estadística. Preddvor, Eslovenia. Septiembre de 1999.

Fecha de recepción: 20 de enero, 2015 Fecha de recepción de la versión modificada: 19 de abril, 2015 Fecha de aceptación: 11 de junio, 2015 